\title{
Prefrontal and Accumbal Electric Activity during Auditory Stimulation in Virgin Female Rats: Changes Related to the Estrous Cycle
}

\author{
Marisela Hernández-González ${ }^{1 *}$, Diego Radberto Chapa García Abascal ${ }^{1}$, Miguel Ángel Guevara ${ }^{1}$, \\ Ricardo Alonso Romero Orozco ${ }^{1}$, Mayra Linné Almanza Sepúlveda ${ }^{1}$, \\ Leopoldo Eduardo Flores Mancilla ${ }^{2}$ \\ ${ }^{1}$ Instituto de Neurociencias, Universidad de Guadalajara, Guadalajara, México \\ ${ }^{2}$ Facultad de Medicina, Universidad Autónoma de Zacatecas, Unidad Académica de Medicina Humana y Ciencias de la Salud, \\ Zacatecas, México \\ Email: *mariselh@cencar.udg.mx
}

Received August 16, 2013; revised September 5, 2013; accepted September 15, 2013

Copyright (C) 2013 Marisela Hernández-González et al. This is an open access article distributed under the Creative Commons Attribution License, which permits unrestricted use, distribution, and reproduction in any medium, provided the original work is properly cited.

\begin{abstract}
The estrous cycle is a physiological process modulated by hormonal changes associated, in turn, with alterations of the attraction or sensitivity with which females perceive different sensory stimuli. Auditory stimuli play an important role in the social interaction of several mammals, including their sexual behavior and the mother-young relationship. Since the prefrontal cortex (PFC) and nucleus accumbens (Acc) had been associated with the processing and assignation of the incentive value of stimuli, this study was designed to analyze whether electroencephalographic (EEG) activity in the PFC and Acc was modified during the two antagonist phases of the estrous cycle (proestrus-estrus and diestrus) when female rats heard sound recordings with different types of cries emitted by other rats. EEGs were recorded in eight female virgin Wistar rats, 80 - 100 days old, bilaterally implanted in the PFC and Acc during an awake-quiet state, in three conditions: when hearing 1) cries of pups; 2) cries of adult male rats; and, 3) cries of adult female rats. The cries were recorded from pups ( 2 - 10 days old $)$ and male and female adult rats after gentle prodding by a human hand. Each auditory stimulus (which included vocalizations up to $22 \mathrm{kHz}$ ) was randomly played back through a speaker placed near the female rats. Only upon perceiving the cries of male rats did the females in the proestrus-estrous phase show a lower relative power in the $14-30 \mathrm{~Hz}$ band in the Acc and an increased inter-prefrontal correlation in the $8-13 \mathrm{~Hz}$ band. It is probable that these EEG changes are associated with the lower activation or non-anxious state that the female rat presents specifically during the proestrus-estrous phase in response to the alarm cries of adult male rats.
\end{abstract}

Keywords: Auditory Stimulation; EEG; Accumbens; Prefrontal Cortex; Estrous Cycle; Rats

\section{Introduction}

The different reproductive phases of the female rat (estrous cycle, pregnancy, and lactation) are associated with hormonal, cognitive, emotional and behavioral changes, as well as variations in sensorial sensitivity. The estrous cycle is a physiological process modulated by hormonal changes associated with alterations in the attraction or sensitivity with which females perceive different sensory stimuli $[1,2]$. Perception of sensory stimuli, especially those of an olfactory and somatosensory nature [1,2], anxiety levels [3], the willingness of cycling female rats

${ }^{*}$ Corresponding author. to show maternal behavior [4], pain thresholds [5] and the excitability and electrical activity of several brain structures $[2,6,7]$ are known to vary across the estrous cycle.

Several studies suggest that in many species the physiological and behavioral output of the auditory system is modulated by hormonal cycles; for example, the perception of behaviorally-relevant auditory signs in mice is affected by hormonal status $[8,9]$. Similarly, subtle changes in auditory perception during the menstrual cycle in women have been suggested [10], especially more acute auditory sensitivity in the periovulatory phase $[11,12]$. Also, the most pronounced menstrual cycle-related changes in audi- 
tory event-related potentials occur in the luteal phase [13].

Auditory stimuli play an important role in the social interaction of several rodents, including their sexual behavior and the mother-young relationship, such that these species-specific vocalizations are used to communicate information related to identity (individual or group), mood (fear or aggression), their next likely behavior (approach, flight, playing, grooming, or mounting), and environmental conditions (presence of predators or location of food). The transmission of different types of information through vocalizations depends on the specific frequency and temporal properties of the acoustic signals $[14,15]$. Three classes of ultrasonic vocalizations (USVs) (inaudible to humans, $>20 \mathrm{kHz}$ ) and one sonic vocalization (audible to humans, $<20 \mathrm{kHz}$ ) have been described in laboratory rats $[16,17]$. When isolated from the dam, pup rats emit vocalizations that can reach a peak frequency of $40 \mathrm{kHz}$ [18], whereas adult rats emit two distinct USVs: one at $50 \mathrm{kHz}$ that tends to be produced in non-aggressive conspecific social interactions [19], during play [20] and in the male's ejaculatory period [21], and the other at 22 $\mathrm{kHz}$ that includes cries emitted during post-ejaculation $[22,23]$, in intraspecific defensive/submissive postures as part of inter-male social interaction [24-26], and upon exposure to a predator [27] or physical distress [28].

In recent years, substantial evidence has accumulated indicating the critical role of the PFC in mediating the assignation of the incentive value of stimuli, and it has been suggested that it is in this cortical region, together with other subcortical areas such as the accumbens nucleus (Acc), that the sensory inputs generated by relevant distant stimuli are processed [29-31]. For example, studies have shown that PFC neurons show different responses to conditioned sensory stimuli (auditory or visual) associated, or not, with a reward [32,33], and that in response to the presentation of emotionally-relevant maternal nursing calls, the mPFC of Octodon degus pups manifest an enhanced metabolic activation [34], similar to that of human mothers when they hear an infant's cries [35]. Also, a characteristic electroencephalographic (EEG) pattern of the PFC has been demonstrated when male rats are exposed to the stimuli emitted by a receptive female rat [36]. With respect to the Acc, certain microdialysis studies have indicated that extracellular DA in the Acc increases when male rats are exposed to inaccessible receptive females [37,38]. Moreover, recent studies support the participation of the Acc in sexual arousal: for example, 6-hydroxydopamine or radiofrequency lesions in the Acc resulted in a lower incidence of non-contact erections (NCE), longer latency to display NCE and fewer erections [30], leading to the suggestion that the Acc is involved in responsiveness to conditioned stimuli or remote cues $[39,40]$.

The recording of EEGs from cortical and subcortical structures constitutes a useful tool for relating changes in brain electrical activity to the performance of various behavioral responses [41,42], as well as to different modalities of sensory stimulation $[43,44]$. The EEG, defined as a mixture of rhythmic, sinusoidal-like fluctuations in voltage generated by the brain, represents the global activity of the pyramidal cells of the cortex and of the neurons in the subcortical structures. Thus, quantitative analyses of EEGs have allowed researchers to probe the simultaneous functioning of several brain structures in a precise temporal relation with specific physiological states, behaviors and sensorial processing. One advantage of EEG-recording is that it makes it possible to obtain an index of the functional coupling between two different brain sites, so that by calculating the correlation parameter, it is easy to determine the degree of similarity between the EEG activity from two brain sites [45].

Although numerous studies have been carried out with the aim of determining and characterizing the different types of vocalizations emitted by rats, and the circumstances in which they are produced, no research has yet ascertained how cerebral electrical activity changes in response to auditory stimulation in the receptor rat. Thus, considering that sensorial sensibility changes in relation to the estrous cycle, that the vocalizations emitted by rats play a pivotal role as means of social communication, and that the PFC and Acc participate in the processing of relevant stimuli, the objective of this study is to determine if EEG activity and the correlation of the PFC and Acc change in relation to the two antagonist phases of the estrous cycle - proestrous-estrous (P-E) and diestrous (D) - when female virgin rats perceive the cries of pups, adult male rats, and adult female rats, respectively.

\section{Methods}

\subsection{Subjects}

Female Wistar rats, 80 - 90 days old, were obtained from a colony bred at the Institute of Neurosciences, University of Guadalajara. All females were housed individually in a room at $22^{\circ} \mathrm{C}-23^{\circ} \mathrm{C}$ under a $12: 12 \mathrm{~h}$ reversed light/dark cycle (lights on from 2000 - 0800 h). Food and water were available ad libitum. Temperature, feeding, and the light-dark cycle conditions were maintained constant throughout the course of study. Animal care and all procedures involving the rats were approved by our Institutional Animal Care and Use Committee in accordance with NIH specifications.

\subsection{Surgery}

For surgery, 12 female rats were anaesthetized with sodium pentobarbital (35 mg/kg i.p.). Stainless steel electrodes $(0.2 \mathrm{~mm}$ in diameter $)$ were bilaterally implanted 
simultaneously in both hemispheres, specifically into the prelimbic (cingulated cortex, area $\mathrm{Cg} 3$ ) region of the PFC (3.2 mm anterior to bregma, $0.6 \mathrm{~mm}$ lateral to midline, and $3.2 \mathrm{~mm}$ below the dura mater) and the shell region of the Acc $(2.7 \mathrm{~mm}$ anterior to bregma, $0.8 \mathrm{~mm}$ lateral to midline, $7 \mathrm{~mm}$ below the dura mater) with the incisor bar set at $-3.5 \mathrm{~mm}$, following Paxinos and Watson's stereotaxic atlas [46]. Two stainless steel screws were placed in the anterior and posterior parts of the skull to serve as the "reference" and "ground" electrodes, respectively. All electrodes were attached to a miniature connector fixed to the skull by means of stainless-steel hooks and acrylic cement. Adequate care was taken to minimize pain or discomfort of the animals throughout the experiment. After surgery, all subjects were housed in individual cages with food and water ad libitum.

\subsection{EEG Tests}

All females were allowed to adapt to the recording room (at $22^{\circ} \mathrm{C}-23^{\circ} \mathrm{C}$, illuminated with dim red light) for at least $1 \mathrm{~h}$ before beginning the EEG tests. The auditory stimulation test was performed during the dark phase of the light-dark cycle. The experimental procedure for auditory stimulation was as follows: each female rat was placed in the recording cage $(43 \times 53 \times 20 \mathrm{~cm}$ made of clear Plexiglas with clean sawdust bedding) where she remained for at least $5 \mathrm{~min}$ before testing began. Immediately after that, the EEGs from the left and right PFC and Acc were recorded simultaneously under three different auditory conditions: 1) hearing cries of pups; 2) hearing cries of adult male rats; and, 3) hearing cries of adult female rats. In all conditions, EEGs were recorded while the undisturbed female rat had its eyes open and its four paws beneath her, with no movement except for slight vibrissae motions. Presentation of the auditory stimuli was randomly counterbalanced and the inter-stimuli interval was $5 \mathrm{~min}$. Each auditory stimulus (including vocalizations up to $22 \mathrm{kHz}$ ) was randomly played back through a speaker placed near the female rat. The duration of each auditory stimuli was $3 \mathrm{~min}$., and each rat was tested in two conditions: namely, the P-E and D phases, which were also counterbalanced.

\subsection{Recording of the Auditory Stimuli}

All three types of cries used as auditory stimuli in this experiment (2-10-day-old pups, adult male rats and adult female rats after gentle prodding by a human hand) were recorded in a soundproofed room where an experimenter subjected the rat and then gently touched its hind feet with a gloved hand not more than 2 - 4 times per minute until it emitted audible cries. The cries were detected by a high-frequency microphone (ACO Pacific, Inc. Model
4012) placed at a distance of $10-20 \mathrm{~cm}$ from their heads and connected to a computer (Toshiba Satellite model L510) that recorded the vocalizations on a hard disc in type. wav files at a frequency of $44,100 \mathrm{~Hz}$ (32 bits per sample), using an audio channel.

\subsection{Exposure to the Auditory Stimuli}

During EEG testing, each female rat was placed in the experimental cage together with the computer's speaker (Usap 3520, $2200 \mathrm{~W}$, three-point with a sub-woofer) that reproduced frequencies up to $50 \mathrm{kHz}$. As the animals usually did not move or rear, the distance between the speaker and their heads was maintained at approximately $20-30 \mathrm{~cm}$. Appropriate corrections were made for movement by the rats, the distance to the speaker, and the rat's head position during all EEG recordings.

\subsection{Recording and Sampling of EEG Activity}

For EEG recording, the connection cable from the electrodes implanted on the rat was passed through a hole in the center of the top of a wire cage $(73 \times 57 \times 61 \mathrm{~cm})$ into which the Plexiglas recording cage was placed. This arrangement allowed free movement by the rat inside the recording cage during continuous recording of the EEGs from the PFC and Acc of the two hemispheres. The cable was connected to the $\mathrm{AC}$ preamplifiers of a Grass 7B polygraph (band pass $3-30 \mathrm{~Hz}$ ) and their outputs were plugged into a PCL-812 analog-to-digital converter (Advantech, Co.) that operated as an interface to a microcomputer. The EEG signals were recorded at a sampling rate of $256 \mathrm{~Hz}$ during $2 \mathrm{sec}$ EEG epochs and were calibrated with a pulse of 50 microvolts $(\mu \mathrm{V})$ produced by the preamplifiers and delivered to the $\mathrm{PC}$ as a reference to convert the output from the analog-to-digital converter to $\mu \mathrm{V}$. The capture of the EEG signals that corresponded to each of the three different conditions was performed by means of a control board with eight on/off buttons that was connected to the digital input lines of the analog-to-digital converter. Thus, the capture of $2 \mathrm{sec}$ segments of EEG corresponding to the cries of pups, adult male rats, and adult female rats began when a specific button on the board was pressed; another button was pushed to end signal input.

By means of specific computer programs [47], the capture of the simultaneous bilateral recording of the EEG signals from PFC and Acc was made in a precise temporal relation with each auditory condition. Various EEG segments of $2 \mathrm{sec}$ were captured in each condition and independently stored in files for off-line analysis.

\subsection{EEG Analysis}

All EEG signals were carefully inspected before analysis 
to discard any segment that contained artifacts. At least ten 2-sec EEG segments (maximum 20) from each subject, condition and reproductive state, were analyzed. Relative power (RP, the proportional contribution of each band expressed as a percentage of total power between 4 and $30 \mathrm{~Hz}$ ) was calculated by means of a Fast Fourier Transformation (FFT) for each band analyzed (4 - 7, 8 13 and $14-30 \mathrm{~Hz}$ ). In order to obtain the degree of functional synchronization between bilateral structures, the inter-prefrontal (right PFC with left PFC) and inter-accumbens (right Acc with left Acc) correlations were obtained in the time domain for the same bands by means of Pearson product-moment correlation coefficients. The EEG corresponding to each condition was compared for each reproductive state.

\subsection{Histology}

At the end of each experiment, the animals were deeply anesthetized with sodium pentobarbital. An intracardial infusion of isotonic saline (0.9\%) followed by $5.0 \%$ buffered paraformaldehyde solution fixed the brain, which was then removed and stored in formalin for at least 2 weeks. Sections 50 microns thick were made with a microtome and stained with cresyl violet. Inspection under a stereoscopic microscope following the stereotaxic coordinates allowed the reconstruction of the path followed by the recording electrode. Only those recordings obtained from the prelimbic (cingulated cortex, area $\mathrm{Cg} 3$ ) region of the PFC and the shell region of the Acc were included for data analysis.

\subsection{Statistics}

Statistical analysis was limited to $4-30 \mathrm{~Hz}$, since lower

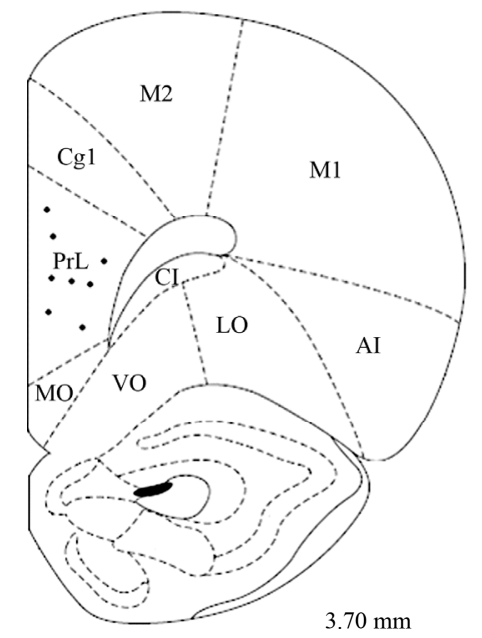

frequencies may include artifacts produced by cable movement. Using a Student t test for correlated groups, the EEG activity of the phases of the estrous cycle in each one of the three conditions (cries of pups, cries of adult male rats, cries of adult female rats) was compared. For statistical purposes, correlation values were transformed to Fisher's Z-scores. Significance was set at $\mathrm{p}<$ 0.05 (two-tailed).

\section{Results}

\subsection{Histology}

The tips of the electrodes were correctly placed in 8 of the 12 female rats; the PFC electrodes were bilaterally located between 3.7 and $3.2 \mathrm{~mm}$ anterior to bregma (in the prelimbic region of the PFC), while the Acc electrodes were placed between 1.2 and $2.2 \mathrm{~mm}$ anterior to bregma (in the shell region of the Acc) (Figure 1).

\subsection{Electroencephalographic Changes}

No significant differences were observed between the two estrous phases in the PFC and Acc when the female rats heard the cries of pups or those of adult female rats. It was only during detection of the cries of adult male rats that significant changes in the RP of the Acc and inter-prefrontal correlation were obtained.

\subsubsection{Relative Power (RP)}

When the females were in the P-E phase, the EEG activity of the Acc was characterized by a decreased RP of the fast frequencies $(14-30 \mathrm{~Hz})$ in both the left $(\mathrm{t}=3.279 ; \mathrm{p}$ $=0.01351)$ and right $(\mathrm{t}=-3.326 ; \mathrm{p}=0.012)$ Acc, compared to when they were in the D phase, but only when exposed to the cries of adult male rats (Figure 2).

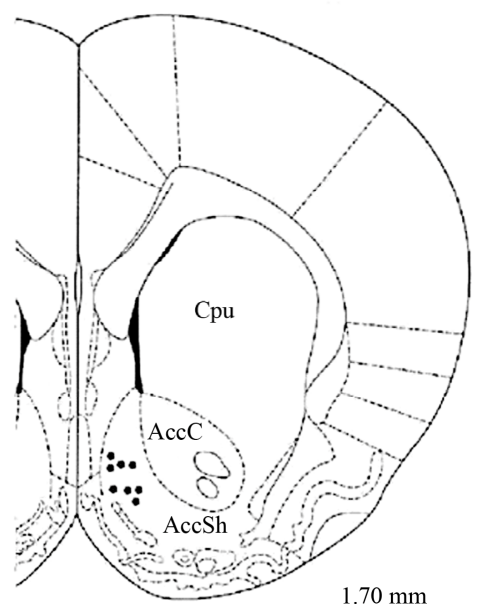

Figure 1. Schematic representation of electrode tip placements in PFC (prelimbic PrL region) and Acc shell region (AccSh) (n = 8). MO, medial orbital cortex; PrL, prelimbic cortex; Cg1, cingulated cortex area; M1, primary motor cortex; M2, secondary motor cortex; LO, lateral orbital cortex; VO, ventral orbital cortex; AI, agranular insular cortex; Pir, piriform cortex; Cpu, caudate-putamen nucleus; AccC, Acc core region. Anterior-posterior coordinates are given with respect to bregma. 


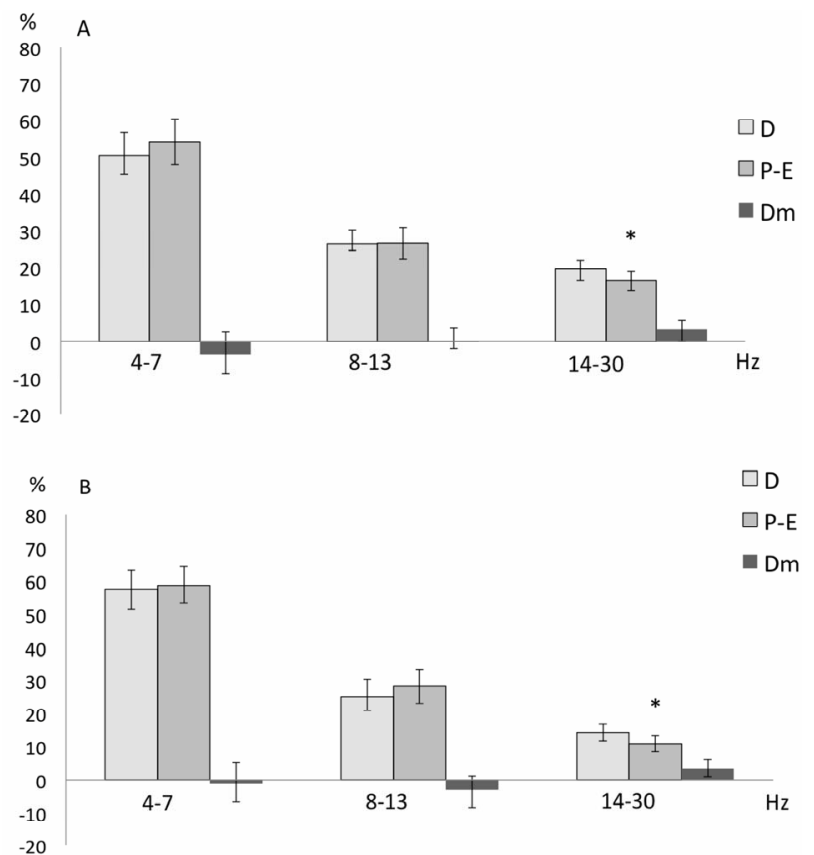

Figure 2. Mean $\pm \mathrm{SE}$ and mean of differences $(\mathrm{Dm})$ of the relative power (in \%) of the three frequency bands recorded in the left (A) and right (B) Accumbens nucleus of female rats during the proestrus-estrus (P-E) and diestrus (D) phases while hearing cries of adult male rats. Student's tests for correlated groups. " $p \leq 0.05$ as compared to $D$ phase.

\subsubsection{Interhemispheric Correlation}

It was only when the female rats were in the P-E phase that the degree of EEG coupling between the left and right PFC was affected by detection of the cries of adult male rats, such that an increased inter-prefrontal correlation of the $8-13 \mathrm{~Hz}$ band was obtained in the P-E phase as compared to the $\mathrm{D}$ phase $(\mathrm{t}=-3.066 ; \mathrm{p}=0.01817)$ (Figure 3).

\section{Discussion}

The results of the present study show that the EEG activity of the Acc and the inter-prefrontal correlation presented characteristic changes specifically in response to the auditory stimuli emitted by adult male rats only in the P-E female rats.

These EEG data agree with other studies which have reported that cerebral functionality changes in relation to estrous phases $[2,6,7,44]$, and that the sensitivity and functioning of the PFC and Acc vary in accordance with both the reproductive state and the auditory stimulation to which female rats are exposed.

Various studies have shown that, like women $[13,48]$, female rats present a relation between the hormonal fluctuations that characterize the estrous cycle and cognitive, perceptual and emotional changes. [3], for example, Fernández Guasti and Picazo [3], reported changes in the

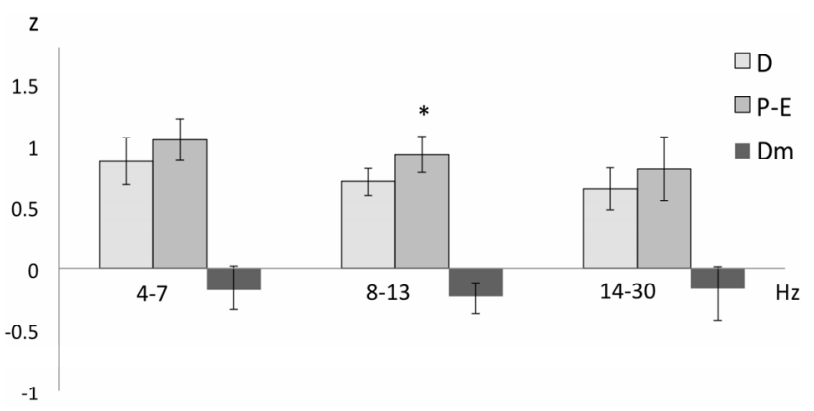

Figure 3. Mean $\pm \mathrm{SE}$ and mean of differences (Dm) of the inter-prefrontal correlation (in z-values) of the three frequency bands recorded in the proestrus-estrus (P-E) and diestrus (D) phases of the female rats while hearing cries of adult male rats. Student's tests for correlated groups. " $p \leq$ 0.05 as compared to the $D$ phase.

anxiety levels of female rats during the different estrous phases, while Pietras and Moulton [1] reported a higher sensitivity to different odors during the estrous phase in rats.

In the present study, a decreased RP of the fast frequencies $(14-30 \mathrm{~Hz})$ in the left and right Acc was observed only when female rats in the P-E phase heard cries of adult male rats. Numerous studies have described irregular, low voltage fast activity (from 12 to over $40 \mathrm{~Hz}$ ) under various behavioral situations of increased alertness [49] or as a response to optimal sensory stimuli, so that these fast frequencies have been considered an index of general activation in animals and humans [50]. On the other hand, the Shell region of the Acc has been shown to play an important role in the processing of stimuli emitted at distance; for example, 6-hydroxydopamine or radiofrequency lesions in the Acc resulted in a lower incidence of non-contact erections (NCE), longer latency to display NCE and fewer erections [30], findings that support the hypothesized role of the Acc in responding to remote cues from estrous females [51]. Fluctuations in circulating hormonal levels during the menstrual cycle are also associated with changes in mood and anxiety [52]. In the rat, anxiety-evaluated by the burying behavior test-decreases during P-E [3], indicating that estrous rats are less anxious than $\mathrm{D}$ rats [53]. Thus, the fact that the Acc of the P-E rats showed a decreased percentage of fast activity when they were exposed to the cries of male rats could be associated with the non-anxious state of those rats. In the D rats, in contrast, a possible increased arousal similar to an anxiety state could be reflected by the high proportion of fast frequencies, an idea that might be supported by the fact that, according to experimental testing, ovariectomized rats or those in metoestrus or diestrus show higher levels of anxiety compared to females in the late proestrus or estrus phases [3].

The degree of $r$ between two neural regions is indicated by the degree of similarity between the EEG activ- 
ity at the two sites [45], which in turn is the outcome of the functional states of the neuronal networks [54]. Low $r$ reflects higher functional differences and vice versa: i.e., higher $\mathrm{r}$ reflects a more homogeneous functioning between structures. The inter-prefrontal $r$ between the left and right PFC showed a different pattern depending on the condition and reproductive state of the rat. In general terms, when the females in the P-E phase heard the cries of male adult rats, higher $r$ values in the $8-12 \mathrm{~Hz}$ band were obtained. A predominance of low frequencies (6 $12 \mathrm{~Hz}$ ) has been related to spatial orientation, voluntary movements and motivated tasks, as well as to motiveemotional conditions during alert immobility in rats [55]. Similarly, a higher proportion and inter-prefrontal correlation at these low frequencies have been reported in sexually-motivated male rats placed near an inaccessible receptive female rat [36]. The fact that during the P-E phase a higher correlation between the prefrontal cortices was obtained in association with the cries of adult male rats does not indicate a higher degree of participation in the processing of this stimuli, but simply reflects a characteristic coupling in the functionality of the prefrontal areas in relation to the aroused condition that exists when the female rat perceives remote stimuli from an adult male rat.

In this study, the female rats heard recordings of cries that included both sonic $(<20 \mathrm{KHz})$ and ultrasonic $(20$ $22 \mathrm{kHz}$ ) vocalizations, so that it is difficult to separate and determine the types of vocalizations that were included in the auditory stimuli and, hence, their emotional meaning. Earlier studies have described that rats emit 22 $\mathrm{kHz}$ USV in very different circumstances, including the post-ejaculatory period $[22,23]$, while engaging in intraspecific defensive/submissive postures as part of intermale social interaction [24-26], when exposed to a predator [27], and in a model of chronic pain [56]. As a result, 22-kilohertz USVs have been suggested as a measure of affective shifts in rats $[20,57]$ and used in a variety of conditioned and unconditioned models of anxiety [58-60]. Sonic vocalization occurs in rats as part of a defensive threat response to an oncoming predator [16]; though less prominent in laboratory rats, it can be elicited by rough handling [28], leading to the suggestion that these audible vocalizations (including the $22 \mathrm{kHz}$ ) may play an adaptive role in increasing chances of survival by serving as indicators of alarm. A study by Brudzynski and Ociepa [28] demonstrated that even a very light touch by the human hand, especially on certain areas of the rat's body, can evoke repeatable ultrasonic vocalizations in the 22 $\mathrm{KHz}$ range comparable to those reported in other situations. Thus, considering that the elevated estrogen levels of the P-E phase have been associated with lower anxiety levels, it is probable that the female rats in this phase interpret the cries of the males only as a novel signal and not as a potential danger, and could therefore be associated with the lower proportion of fast frequencies in the Acc nucleus and the higher inter-prefrontal correlation.

Although there is abundant information on the conditions and, hence, possible meanings of the USV emitted by rats $[17-19,23,27,28,58,61,62]$, little knowledge exists on the effect that cries in the audible range induce in the behavior and brain functionality of the receptor rat. It is well known that male rats emit USV in the range of 22 $\mathrm{KHz}$ under novel or distressful conditions [18] and during the post-ejaculatory interval $[22,23]$. Though it is very difficult to determine the specific types of vocalizations that were present in the auditory stimuli used in this work, the EEG data show that it was only during the P-E phase that the cries of adult male rats affected the functionality of the PFC and Acc; a finding that was not observed when the female rats heard the cries of pups or adult females. Therefore, it is probable that the lower proportion of the fast frequencies in the Acc and the higher inter-prefrontal correlation in the $8-13 \mathrm{~Hz}$ band represent a characteristic EEG pattern that could be associated with the attention and/or non-anxious state of the female rats in the P-E phase. Another possible explanation is that considering that the auditory stimuli used in this experiment included frequencies up to $22 \mathrm{KHz}$ (which are emitted during the post-ejaculatory interval), and that it is precisely in the P-E phase that the female rat is more sensitive to cues emitted by males, it is probable that the estrous females could interpret the cries emitted by adult males as a mating call.

Thus, the EEG data presented in this study support the suggestion that, as earlier research has proposed [29-31, 51], the PFC and Acc could participate in the processing of, and assignation of incentive value to, auditory stimuli in estrous female rats. It is probable that given the elevated estrogen levels of the female rats in this phase they could interpret the cries of the males as a novel signal or mating call and not as a sign of potential danger.

\section{REFERENCES}

[1] J. R. Pietras and G. D. Moulton, "Hormonal Influences on Odor Detection in Rats: Changes Associated with the Estrous Cycle, Pseudopregnancy, Ovariectomy, and Administration of Testosterone Propionate," Physiology \& Behavior, Vol. 12, 1974, pp. 475-491.

http://dx.doi.org/10.1016/0031-9384(74)90125-5

[2] R. Guevara-Guzmán, B. Barrera-Mera and M. L. Weiss, "Effect of the Estrous Cycle on Olfactory Bulb Response to Vaginocervical Stimulation in the Rat: Results from Electrophysiology and Fos Immunicytochemistry Experiments," Brain research bulletin, Vol. 44, No. 2, 1997, pp. 141-149. http://dx.doi.org/10.1016/S0361-9230(97)00103-2

[3] A. Fernandez-Guasti and O. Picazo, "Changes in Burying Behavior during the Estrous Cycle: Effect of Estrogen 
and Progesterone," Psychoneuroendocrinology, Vol. 17, No. 6, 1992, pp. 681-689.

http://dx.doi.org/10.1016/0306-4530(92)90027-5

[4] D. E. Gonzalez and R. P. Deis, "Maternal Behavior in Cyclic and Androgenized Female Rats. Role of Ovarian Hormones," Physiology \& Behavior, Vol. 38, No. 8, 1986, pp. 789-793.

http://dx.doi.org/10.1016/0031-9384(86)90044-2

[5] M. Martínez-Gómez, Y. Cruz, M. Salas, R. Hudson and P. Pacheco, "Assessing Pain Threshold in the Rat: Changes with Estrus and Time of Day," Physiology \& Behavior, Vol. 55, No. 4, 1994, pp. 651-657. http://dx.doi.org/10.1016/0031-9384(94)90040-X

[6] M. Corsi-Cabrera, J. Juarez, M. Ponce-de-Leon, J. Ramos and P. N. Velásquez, "EEG Activity during Estral Cycle in the Rat," Electroencephalography and Clinical Neurophysiology, Vol. 83, No. 4, 1992, pp. 265-269. http://dx.doi.org/10.1016/0013-4694(92)90120-7

[7] C. M. Contreras, M. Molina, M. Saavedra and L. Martínez-Mota, "Lateral Septal Neuronal Firing Rate Increase during Proestrus-Estrus in the Rat," Physiology \& Behavior, Vol. 68, No. 3, 2000, pp. 279-284.

http://dx.doi.org/10.1016/S0031-9384(99)00169-9

[8] G. Ehret and C. Schmid, "Reproductive Cycle-Dependent Plasticity of Perception of Acoustic Meaning in Mice," Physiology \& Behavior, Vol. 96, No. 3, 2009, pp. 428433. http://dx.doi.org/10.1016/i.physbeh.2008.11.005

[9] J. A. Miranda and R. C. Liu, "Dissecting Natural Sensory Plasticity: Hormones and Experience in a Maternal Context," Hearing Research, Vol. 252, No. 1-2, 2009, pp. 2128. http://dx.doi.org/10.1016/j.heares.2009.04.014

[10] M. Haggard and J. B. Gaston, "Changes in Auditory Perception in the Menstrual Cycle," British Journal of Audiology, Vol. 12, No. 4, 1978, pp. 105-118. http://dx.doi.org/10.3109/03005367809078862

[11] D. Al-Mana, B. Ceranic, O. Djahanbakhch and L. M. Luxon, "Alteration in Auditory Function during the Ovarian Cycle," Hearing Research, Vol. 268, No. 1-2, 2010, pp. 114-122. http://dx.doi.org/10.1016/j.heares.2010.05.007

[12] A. Serra, L. Maiolino, C. Agnello, A. Messina and S. Caruso, "Auditory Brain Stem Response throughout the Menstrual Cycle," The Annals of Otology, Rhinology, and Laryngology, Vol. 112, No. 6, 2003, pp. 549-553.

[13] V. Walpurger, R. Pietrowsky, C. Kirschbaum and O. T. Wolf, "Effects of the Menstrual Cycle on Auditory EventRelated Potentials," Hormones and Behavior, Vol. 46, No. 5, 2004, pp. 600-606. http://dx.doi.org/10.1016/j.yhbeh.2004.07.002

[14] D. B. Geissler and G. Ehret, "Time-Critical Integration of Formants for Perception of Communication Calls in Mice," Proceedings of the National Academy of Sciences of the United States of America, Vol. 99, No. 13, 2002, pp. 9021-9025. http://dx.doi.org/10.1073/pnas.122606499

[15] N. Suga, "Philosophy and Stimulus Design for Neuroethology of Complex-Sound Processing," Philosophical Transactions of the Royal Society of London. Series B, Biological Sciences, Vol. 336, No. 1278, 1992, pp. 423-428. http://dx.doi.org/10.1098/rstb.1992.0078
[16] R. J. Blanchard, K. J. Flannelly and D. C. Blanchard, "Defensive Behavior of Laboratory and Wild Rattus norvegicus," Journal of Comparative Psychology, Vol. 100, No. 2, 1986, pp. 101-107. http://dx.doi.org/10.1037/0735-7036.100.2.101

[17] S. M. Brudzynski, F. Bihari, D. Ociepa and X. W. Fu, "Analysis of $22 \mathrm{kHz}$ Ultrasonic Vocalization in Laboratory Rats: long and Short Calls," Physiology \& Behavior, Vol. 54, No. 2, 1993, pp. 215-221. http://dx.doi.org/10.1016/0031-9384(93)90102-L

[18] S. M. Brudzynski, P. Kehoe and M. Callahan, "Sonographic Structure of Isolationinduced Ultrasonic Calls of Rat Pups," Developmental Psychobiology, Vol. 34, No. 3, 1999, pp. 195-204.

[19] R. J. Blanchard, E. B. Yudko, D. C. Blanchard and H. K. Taukulis, "High-Frequency (35-70 kHz) Ultrasonic Vocalizations in Rats Confronted with Anesthetized Con- specifics: Effects of Gepirone, Ethanol, and Diazepam," Pharmacology Biochemistry and Behavior, Vol. 44, No. 2, 1993, pp. 313-319.

http://dx.doi.org/10.1016/0091-3057(93)90467-8

[20] B. Knutson, J. Burgdorf and J. Panksepp, “Anticipation of Play Elicits High Frequency Ultrasonic Vocalizations in Young Rats," Journal of Comparative Psychology, Vol. 112, No. 1, 1998, pp. 65-73.

http://dx.doi.org/10.1037/0735-7036.112.1.65

[21] T. K. McIntosh and R. J. Barfield, "The Temporal Patterning of 40-60 kHz Ultrasonic Vocalizations and Copulation in the Rat (Rattus norvegicus)," Behavioral and Neural Biology, Vol. 29, No. 3, 1980, pp. 349-358. http://dx.doi.org/10.1016/S0163-1047(80)90259-9

[22] R. J. Barfield and L. A. Geyer, "Sexual Behavior: Ultrasonic Postejaculatory Song of the Male Rat," Science, Vol. 176, No. 4041, 1972, pp. 1349-1350. http://dx.doi.org/10.1126/science.176.4041.1349

[23] R. J. Barfield and D. A. Thomas, "The Role of Ultrasonic Vocalizations in the Regulation of Reproduction in Rats," Annals of the New York Academy of Sciences, Vol. 474, 1986, pp. 33-43. http://dx.doi.org/10.1111/j.1749-6632.1986.tb27996.x

[24] R. Lore, K. Flannelly and P. Farina, "Ultrasounds Produced by Rats Accompany Intraspecific Fighting," Aggressive Behavior, Vol. 2, No. 3, 1976, pp. 175-181.

[25] M. Portavella, A. Depaulis and M. Vergnes, "22-28 kHz Ultrasonic Vocalizations Associated with Defensive Reactions in Male Rats Do Not Result from Fear or Aversion," Psychopharmacology, Vol. 111, No. 2, 1993, pp. 190-194. http://dx.doi.org/10.1007/BF02245522

[26] D. A. Thomas, L. K. Takahashi and R. J. Barfield, "Analysis of Ultrasonic Vocalizations Emitted by Intruders during Aggressive Encounters among Rats (Rattus norvegicus)," Journal of Comparative Psychology, Vol. 97, No. 3, 1983, pp. 201-206. http://dx.doi.org/10.1037/0735-7036.97.3.201

[27] R. J. Blanchard, D. C. Blanchard, R. Agullana and S. M. Weiss, "Twenty-Two kHz Alarm Cries to Presentation of a Predator, by Laboratory Rats Living in Visible Burrow Systems," Physiology \& Behavior, Vol. 50, No. 5, 1991, pp. 967-972. 
http://dx.doi.org/10.1016/0031-9384(91)90423-L

[28] S. M. Brudzynski and D. Ociepa, "Ultrasonic Vocalization of Laboratory Rats in Response to Handling and Touch," Physiology \& Behavior, Vol. 52, No. 4, 1992, pp. 655-660. http://dx.doi.org/10.1016/0031-9384(92)90393-G

[29] A. Ågmo, A. Villalpando, Z. Picker and H. Fernández, "Lesions of the Medial Prefrontal Cortex and Sexual Behavior in the Male Rat," Brain Research, Vol. 696, No. 1-2, 1995, pp. 177-186. http://dx.doi.org/10.1016/0006-8993(95)00852-H

[30] Y. C. Liu, B. D. Sachs and J. D. Salamone, "Sexual Behavior in Male Rats after Radiofrequency or DopamineDepleting Lesions in Nucleus Accumbens," Pharmacology, Biochemistry, and Behavior, Vol. 60, No. 2, 1998, pp. 585-592. http://dx.doi.org/10.1016/S0091-3057(98)00022-7

[31] M. Hernández-González and M. A. Guevara, "Participation of the Prefrontal Cortex in the Processing of Sexual and Maternal Incentives," In: L. LoGrassso and G. Morretti, Eds., Prefrontal Cortex: Roles, Interventions and Traumas, Nova Science Publishers, New York, 2009, pp. 115-157.

[32] K. Takenouchi, H. Nishijo, T. Uwano, R. Tamura, M. Takigawa and T. Ono, "Emotional and Behavioral Correlates of the Anterior Cingulated Cortex during Associative Learning in Rats," Neuroscience, Vol. 93, No. 4, 1999, pp. 1271-1287. http://dx.doi.org/10.1016/S0306-4522(99)00216-X

[33] E. Jodo, Y. Suzuki and Y. Kayama, "Selective Responsiveness of Medial Prefrontal Cortex Neurons to the Meaningful Stimulus with a Low Probability of Occurrence in Rats," Brain Research, Vol. 856, No. 1-2, 2000, pp. $68-74$.

http://dx.doi.org/10.1016/S0006-8993(99)02386-0

[34] K. Braun and G. Poeggel, "Recognition of Mother's Voice Evokes Metabolic Activation in the Medial Prefrontal Cortex and Lateral Thalamus of Octodon degus Pups," Neuroscience, Vol. 103, No. 4, 2001, pp. 861-864. http://dx.doi.org/10.1016/S0306-4522(01)00074-4

[35] J. P. Lorberbaum, J. D. Newman, A. R. Horwitz, J. R. Dubno, R. Bruce Lydiard, M. B. Hamner, D. E. Bohning and M. S. George, "A Potential Role for Thalamocingulate Circuitry in Human Maternal Behavior," Biological Psychiatry, Vol. 51, No. 6, 2002, pp. 431-445. http://dx.doi.org/10.1016/S0006-3223(01)01284-7

[36] M. Hernández-González, C. A. Prieto-Beracoechea, M. Arteaga-Silva and M. A. Guevara, "Different Functionality of the Medial and Orbital Prefrontal Cortex during a Sexually Motivated Task in Rats," Physiology \& Behavior, Vol. 90, No. 2-3, 2007, pp. 450-458. http://dx.doi.org/10.1016/j.physbeh.2006.10.006

[37] G. Damsma, J. G. Pfaus, D. G. Wenktern, A. G. Phillips, H. C. Fibiger, "Sexual Behavior Increases Dopamine Transmission in the Nucleus Accumbens and Striatum of Male Rats: Comparison with Novelty and Locomotion," Behavioral Neuroscience, Vol. 106, No. 1, 1992, pp. 181191. http://dx.doi.org/10.1037/0735-7044.106.1.181

[38] J. G. Pfaus, G. Damsma, G. G. Nomikos, D. G. Wenk- stern, C. D. Blaha, A. G. Phillips and H. C. Fibiger, "Sexual Behavior Enhances Central Dopamine Transmission in the Male Rat," Brain Research, Vol. 530, No. 2, 1990 , pp. 345-348.

http://dx.doi.org/10.1016/0006-8993(90)91309-5

[39] M. Cador, J. Taylor and T. Robbins, "Potentiation of the Effects of Reward-Related Stimuli by Dopaminergic-Dependent Mechanisms in the Nucleus Accumbens," Psychopharmacology, Vol. 104, No. 3, 1991, pp. 377-385. http://dx.doi.org/10.1007/BF02246039

[40] J. Salamone, M. Cousins and B. Snyder, "Behavioral Functions of Nucleus Accumbens Dopamine: Empirical and Conceptual Problems with the Anhedonia Hypothesis," Neuroscience and Biobehavioral Reviews, Vol. 21, No. 3, 1997, pp. 341-359. http://dx.doi.org/10.1016/S0149-7634(96)00017-6

[41] L. A. Mead and C. H. Vanderwolf, "Hippocampal Electrical Activity in the Female Rat: The Estrous Cycle, Copulation, Parturition, and Pup Retrieval," Behavioral Brain Research, Vol. 50, No. 1-2, 1992, pp. 105-113. http://dx.doi.org/10.1016/S0166-4328(05)80292-X

[42] M. Hernández-González, M. A. Guevara, M. Cervantes, G. Moralí and M. Corsi-Cabrera, "Characteristic Frequency Bands of the Cortico-Frontal EEG during the Sexual Interaction of the Male Rat as a Result of Factorial Analysis," Journal of Physiology-Paris, Vol. 92, No. 1, 1998, pp. $43-50$. http://dx.doi.org/10.1016/S0928-4257(98)80022-3

[43] P. H. Boeijinga and F. H. Lopez da Silva, "Modulations of EEG Activity in the Entorhinal Cortex and Forebrain Olfactory Areas during Odour Sampling," Brain Research, Vol. 478, No. 2, 1989, pp. 257-268. http://dx.doi.org/10.1016/0006-8993(89)91506-0

[44] M. Hernández-González, C. A. Prieto-Beracoechea, M. Navarro-Meza and M. A. Guevara, "Prefrontal and Tegmental Electrical Activity during Olfactory Stimulation in Virgin and Lactating Rats," Physiology \& Behavior, Vol. 83, No. 5, 2005, pp. 749-758. http://dx.doi.org/10.1016/j.physbeh.2004.09.013

[45] F. Lopes da Silva, "Neural Mechanisms Underlying Brain Waves: From Neural Membranes to Networks," Electroencephalography and Clinical Neurophysiology, Vol. 79, No. 2, 1991, pp. 81-93.

http://dx.doi.org/10.1016/0013-4694(91)90044-5

[46] G. Paxinos and C. H. Watson, "The Rat Brain in Stereotaxic Coordinates," 5th Edition, Elsevier Academic Press, San Diego, 2005.

[47] M. A. Guevara, J. Ramos, M. Hernández-González, H. Madera and M. Corsi-Cabrera, "CAPTUSEN: A System for the Computerized Acquisition of EEG and Event-Related Potentials," Mexican Journal of Psychology, Vol. 17, No. 1, 2000, pp. 77-88.

[48] T. Hummel, R. Gollisch, G. Wildt and G. Kobal, "Changes in Olfactory Perception during the Menstrual Cycle," Experientia, Vol. 47, No. 7, 1991, pp. 712- 715. http://dx.doi.org/10.1007/BF01958823

[49] V. N. Murthy and E. E. Fetz, "Coherent 25 to 35 Hz Oscillations in the Sensorimotor Cortex of Awake Behaving Monkeys," Proceedings of the National Academy of Scien- 
ces of the United States of America, Vol. 89, No. 12, 1992 , pp. 5670-5674.

http://dx.doi.org/10.1073/pnas.89.12.5670

[50] J. T. Coull, "Neural Correlates of Attention and Arousal from Electrophysiology, Functional Neuroimaging and Psychopharmacology," Progress in Neurobiology, Vol. 55, No. 4, 1998, pp. 343-361. http://dx.doi.org/10.1016/S0301-0082(98)00011-2

[51] D. Wenkstern, J. Pfaus and H. Fibiger, "Dopamine Transmission Increases in the Nucleus Accumbens of Male Rats during Their First Exposure to Sexually Receptive Female Rats," Brain Research, Vol. 618, No. 1, 1993, pp. 41-46. http://dx.doi.org/10.1016/0006-8993(93)90426-N

[52] S. H. M. Van Goozen, N. H. Frijda, V. M. Wiegant, E. Endert and N. E. Van de Poll, "The Premenstrual Phase and Reactions to Aversive Events: A Study of Hormonal Influences on Emotionality," Psychoneuroendocrinology, Vol. 21, No. 5, 1996, pp. 479-497. http://dx.doi.org/10.1016/0306-4530(95)00022-4

[53] J. F. Rodriguez-Sierra, J. L. Howard, G. T. Pollard and S. E. Hendriks, "Effect of Ovarian Hormones on Conflict Behavior," Psychoneuroendocrinology, Vol. 9, No. 3, 1984, pp. 293-300. http://dx.doi.org/10.1016/0306-4530(84)90008-8

[54] J. C. Shaw, "Correlation and Coherence Analysis of the EEG: A Selective Tutorial Review," International Journal of Psychophysiology: Official Journal of the International Organization of Psychophysiology, Vol. 1, No. 3, 1984, pp. 255-266.

http://dx.doi.org/10.1016/0167-8760(84)90045-X

[55] R. Kramis, C. H. Vanderwolf and B. H. Bland, "Two Types of Hippocampal Rhythmical Slow Activity in Both the Rabbit and the Rat: Relations to Behavior and Effects of Atropine, Diethyl Ether, Urethane, and Pentobarbital," Experimental Neurology, Vol. 49, No. 1, 1975, pp. 58-85. http://dx.doi.org/10.1016/0014-4886(75)90195-8

[56] B. Calvino, J. M. Besson, A. Boehrer and A. Depaulis, "Ultrasonic Vocalization (22 - $28 \mathrm{kHz})$ in a Model of Chronic Pain, the Arthritic Rat: Effects of Analgesic Drugs,' Neuroreport, Vol. 7, No. 2, 1996, pp. 581-584. http://dx.doi.org/10.1097/00001756-199601310-00049

[57] R. W. Bell, "Ultrasounds in Small Rodents: Arousal-Produced and Arousal Producing," Developmental Psychobiology, Vol. 7, No. 1, 1974, pp. 39-42. http://dx.doi.org/10.1002/dev.420070107

[58] S. M. Brudzynski and E. M. Chiu, "Behavioural Responses of Laboratory Rats to Playback of $22 \mathrm{khz}$ Ultrasonic Calls," Physiology \& Behavior, Vol. 57, No. 6, 1995, pp. 1039-1044. http://dx.doi.org/10.1016/0031-9384(95)00003-2

[59] M. L. Nunes Mamede Rosa, M. J. Nobre, A. Ribeiro Oliveira and M. L. Brandao, "Isolation-Induced Changes in Ultrasonic Vocalization, Fear-Potentiated Startle and Prepulse Inhibition in Rats," Neuropsychobiology, Vol. 51, No. 4, 2005, pp. 248-255. http://dx.doi.org/10.1159/000085820

[60] C. Sanchez, "Stress-Induced Vocalization in Adult Animals. A Valid Model of Anxiety?" European Journal of Pharmacology, Vol. 463, No. 1-3, 2003, pp. 133-143. http://dx.doi.org/10.1016/S0014-2999(03)01277-9

[61] N. Takahashi, M. Kashino and N. Hironaka, "Structure of Rat Ultrasonic Vocalizations and Its Relevance to Behavior," PLOS ONE, Vol. 5, No. 11, 2010. http://dx.doi.org/10.1371/journal.pone.0014115

[62] E. M. S. Snoeren and A. Ågmo, "Female Ultrasonic Vocalizations Have No Incentive Value for Male Rats," Behavioral Neuroscience, Vol. 127, No. 3, 2013, pp. 439-450. http://dx.doi.org/10.1037/a0032027 